\title{
Ensuring Certain Physical Properties in Black Box Models by Applying Fuzzy Techniques
}

\author{
Peter Lindskog and Lennart Ljung \\ Department of Electrical Engineering \\ Linköping University, S-581 83 Linköping, Sweden \\ www: http://www.control.isy.liu.se \\ email: lindskog@isy.liu.se, ljung@isy.liu.se
}

1997-04-10

Conference: SYSID '97

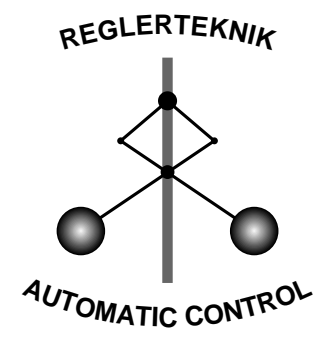

LINKÖPING

Technical reports from the Automatic Control group in Linköping are available by anonymous ftp at the address 130.236.20.24 (ftp.control.isy.liu.se). This report is contained in the compressed postscript file LiTH-ISY-R-1942.ps.Z. 


\title{
ENSURING CERTAIN PHYSICAL PROPERTIES IN BLACK BOX MODELS BY APPLYING FUZZY TECHNIQUES
}

\author{
P. Lindskog* and L. Ljung* \\ * Dept. of Electrical Engineering, Linköping University, Sweden, \\ E-mail: lindskog@isy. liu.se, ljung@isy.liu.se
}

\begin{abstract}
We consider the situation where a nonlinear physical system is identified from input-output data. In case no specific physical structural knowledge about the system is available, parameterized grey box models cannot be used. Identification in black-box-type of model structures is then the only alternative, and general approaches like neural nets, neuro-fuzzy models, etc., have to be applied.

However, certain non-structural knowledge about the system is sometimes available. It could be known, e.g., that the step response is monotonic, or that the steadystate gain curve is monotonic. The question is then how to utilize and maintain such knowledge in a black box framework.

In this paper we show how to incorporate this type of prior information in an otherwise black box environment, by applying a specific fuzzy model structure, with strict parametric constraints. The usefulness of the approach is illustrated by experiments on real-world data.
\end{abstract}

Keywords: Fuzzy modeling and identification; nonlinear systems; monotonicity.

\section{INTRODUCTION}

Don't estimate what you already know!

This is a basic principle in estimation and identification and is also a pragmatic variant of the principle of parsimony - to be parsimonious with parameters to estimate. In an identification context, the concept of grey boxes has been introduced to denote model structures that use some kind of prior information about the system. The term tailor-made model structure has also been used. This is of course in contrast to black boxes or ready-made model structures, which just use "size" as the basic structure option.

Now, there are several shades of grey. Often grey boxes employ rather specific knowledge of the system; as an extreme it may correspond to a complete physical parameterization having some unknown parameters. These parameters are typically estimated by maximum likelihood/prediction error techniques.

The other end of this scale - the black box model structure-uses in the general nonlinear case a function series expansion like

$$
\hat{y}(t \mid \theta)=g(\varphi(t), \theta)=\sum_{j=1}^{n} \theta_{j} g_{j}(\varphi(t)) .
$$

Here $\hat{y}(t \mid \theta) \in \mathbb{R}$ is the model's predicted value of the output $y(t)$ at time $t$, and $\varphi(t)$ are the regressors (past inputs and outputs) that are used to make the prediction. $\mathrm{g}(\cdot)$ is a general mapping, parameterized by $\theta$, and we may think of $g_{j}(\cdot)$ as basis functions, building up the mapping. In turn, these $g_{j}(\cdot)$ could also be parameterized by $\theta$.

There are many choices of expansions of this particular form. Neural nets, wavelet models, fuzzy models, nearest neighbor models, etc., all fit into this framework, see, e.g., (Sjöberg et al., 1995). An important challenge is here to combine the richness and flexibility of (1) with prior physical knowledge that is not of precise, analytical character. We would thus like to work with boxes that are just a shade lighter than black.

Prior system knowledge of this kind is quite often available. It could be, e.g., that the step response 
is monotonic, or that the steady-state gain curve is monotonic in certain input variables, or some other qualitative property.

It is in general not easy to incorporate such information in conventional grey box parameterized model structures. In this contribution we will show how such properties can be cast into a fuzzy modeling framework. While a linguistic description could be well suited to pin down a qualitative behavior in a fuzzy rule base, it is also true that this knowledge can be annihilated by too flexible a parameterization. It is thus necessary to introduce constraints in the parameterization so that the qualitative behavior is guaranteed.

In Sec. 2 we describe one particular fuzzy model structure as one way to obtain the parameterization (1). The monotonicity of the static gain curve is then investigated in Sec. 3, where it is proved how to ensure such a property within an otherwise flexible parameterization. The ideas are finally tested on some real-world data from a heating process in Sec. 4.

\section{FUZZY MODEL STRUCTURE}

The basic structure of a fuzzy model (or controller) is shown in Fig. 1. At the heart of the matter is the fuzzy rule base $\mathbf{R}$, which consists of a set of linguistic production rules

$$
\mathbf{R}=\left\{R_{1}, R_{2}, \ldots, R_{n}\right\},
$$

here assumed to be of the form

$$
\begin{aligned}
R_{j}: & \operatorname{IF}\left(U_{1} \text { IS } A_{j, 1}\right) \text { AND ... AND }\left(U_{r} \text { IS } A_{j, r}\right) \\
& \operatorname{THEN}\left(Y \text { IS } B_{j}\right),
\end{aligned}
$$

with $A_{1,1}, \ldots, A_{n, r}$ being the linguistic values that can be assigned to the linguistic variables $\mathrm{U}_{1}, \ldots, \mathrm{U}_{\mathrm{r}}$ (regressors), while $\mathrm{B}_{1}, \ldots, \mathrm{B}_{\mathrm{n}}$ denote the linguistic values that can be assigned to $\mathrm{Y}$.

Each linguistic value is characterized by a fuzzy set $A \in \mathbb{U}$, defined as the set of ordered pairs

$$
A=\left\{\left(u, \mu_{A}\left(u, \beta_{A}, \gamma_{A}\right)\right): u \in \mathbb{U}\right\}
$$

where $\mu_{A}\left(u, \beta_{A}, \gamma_{A}\right)$ is called a membership function (MF), carrying an element from $\mathbb{U}$ into a membership value between 0 (no degree of membership) and 1 (full degree of membership). The MF can be any function returning a value in $[0,1]$, e.g., a sigmoid, a Gaussian, a triangular, and so on. The scale and position of such an MF are specified by the parameters $\beta_{A}$ and $\gamma_{A}$, respectively.

There are many ways to mathematically interprete the remaining fuzzy constructs. However, several identification aspects (see, e.g., (Lindskog, 1996)) motivate the use of a singleton fuzzifier, a sup-star based inference mechanism with Mamdani implication and algebraic product for AND, along with a center-of-sums defuzzifier; consult (Driankov et al., 1993) for the definitions. The fuzzy rule base (2) can then be translated into the fuzzy model structure

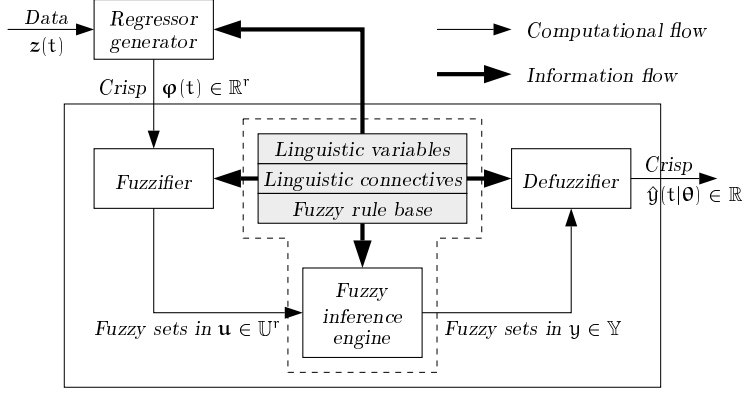

Fig. 1. Basic structure of a fuzzy model.

$$
\begin{array}{r}
\hat{y}(t \mid \theta)=g(\varphi(t), \theta)=\frac{\sum_{j_{1}=1}^{n_{1}} \cdots \sum_{j_{r}=1}^{n_{r}} \alpha_{j} \cdot w_{\mathbf{A}_{j}}}{\sum_{j_{1}=1}^{n_{1}} \cdots \sum_{j_{r}=1}^{n_{r}} w_{\boldsymbol{A}_{j}}} \\
w_{\boldsymbol{A}_{j}}=\prod_{k=1}^{r} \mu_{A_{j_{k}, k}}\left(\varphi_{k}(t), \beta_{j_{k}, k}, \gamma_{j_{k}, k}\right) .
\end{array}
$$

This notation needs some further explanation.

First of all, the overall parameter vector is

$$
\theta^{\top}=\left[\begin{array}{lll}
\boldsymbol{\alpha}^{\top} & \boldsymbol{\beta}^{\top} & \boldsymbol{\gamma}^{\top}
\end{array}\right]
$$

which means that the MFs at the output side (associated with the fuzzy sets $B_{1}, \ldots, B_{n}$ ) are chosen to be fuzzy singletons or centers $\boldsymbol{\alpha}$, whereas the MFs at the input side (associated with the fuzzy sets $A_{1,1}, \ldots, A_{n, r}$ ) involve both scale $\beta$ and position $\gamma$ parameters. This is the same terminology as is suggested in (Sjöberg et al., 1995), where the series expansion (5) is referred to as a tensor product construction. However, contrary to many other similar approaches, it should here be noted that the parameters can be linguistically interpreted. As we shall see in the following section, this is an important property to preserve in the parameter estimation step.

Secondly, a complete fuzzy rule base consists of $n=\prod_{k=1}^{r} n_{k}$ different rules, where $n_{k}$ is the number of MFs describing the kth regressor. Effectively, the labeling in (5) and (6) is just a convenient relabeling of the rules and the corresponding MFs of (2), where $\mathbf{j}=\mathfrak{j}_{1}, \ldots, \boldsymbol{j}_{k}$ is a grid-oriented multi-index, with $j_{k}$ specifying one particular MF associated with the kth regressor. An example of such an enumeration is shown in Fig. 2.

We are now prepared to deal with the problem of how to achieve a monotone steady-state gain curve when applying model structure (5).

\section{ENSURING MONOTONICITY}

Many dynamic processes are known (from physics) to have a steady-state gain curve that is monotonic. Consider, e.g., a simple tank system where the inflow is the input and the liquid level the output. Here it is known that a certain constant inflow eventually leads to a "constant" liquid level. Starting from such a steady-state condition 
we also know that an increase in the inflow causes the liquid level to increase (in a non-oscillatory manner) and settle at a higher level. As will be illustrated in the following section, there are certain applications for which it is crucial that the used models show this kind of monotone behavior.

If we now apply flexible nonlinear model structures (neural nets, etc.), then it can be quite hard to achieve the requested monotonicity, especially if there are regression regions with few and noisy data. To remedy this, we suggest a restricted variant of the fuzzy model structure (5) that guarantees an increasing (decreasing) function mapping from the regression space $\mathbb{R}^{r}$ to the output space $\mathbb{R}$. This structure together with a proper choice of regressor $\varphi(t)$ result in dynamical models having the desired monotone behavior.

A conceptually rather simple way to ensure this property is to first restrict the MFs at the input side to correspond to fuzzy partitions.

Definition 1. (fuzzy partition). Suppose that the $k$ th linguistic variable can be assigned to $n_{k}$ different values each described by a membership function $\mu_{A_{j_{k}, k}}\left(\varphi_{k}(t), \boldsymbol{\beta}_{j_{k}, k}, \gamma_{j_{k}, k}\right) \in \mathbb{U}_{k}$. These MFs form a fuzzy partition if it holds on the entire domain $\mathbb{U}_{\mathrm{k}}$ that

$$
\sum_{j_{k}=1}^{n_{k}} \mu_{A_{j_{k}, k}}\left(\varphi_{k}(t), \beta_{j_{k}, k}, \gamma_{j_{k}, k}\right)=1 \text {. }
$$

By imposing this restriction on all of the $r$ linguistic variables and additionally assuming that the rule base is complete in the sense that it covers the whole input domain $\mathbb{U}^{r}$, it immediately follows that the model structure (5) simplifies to

$$
\hat{\mathrm{y}}(\mathrm{t} \mid \boldsymbol{\theta})=\sum_{\mathbf{j}_{1}=1}^{\mathrm{n}_{1}} \ldots \sum_{\mathbf{j}_{r}=1}^{\mathrm{n}_{r}} \alpha_{\mathbf{j}} \cdot \boldsymbol{w}_{\boldsymbol{A}_{\mathbf{j}}} .
$$

At this point, notice that a fuzzy partition puts certain demands on the MFs and their parameters. For example, we cannot in general use sigmoidal or Gaussian MFs because of their spreading and curvature. Piecewise linear MFs on the other hand can easily be parameterized so that a fuzzy partition is obtained. Within this category we will here restrict the discussion to the open left $\operatorname{mfl}\left(u, \gamma_{1}, \gamma_{2}\right)$, the open right $\operatorname{mfr}\left(u, \gamma_{1}, \gamma_{2}\right)$ and the triangular mftri $\left(u, \gamma_{1}, \gamma_{2}, \gamma_{3}\right) \mathrm{MFs}$, which in order are given by

$$
\begin{aligned}
& \max \left(\min \left(\frac{\gamma_{2}-u}{\gamma_{2}-\gamma_{1}}, 1\right), 0\right), \\
& \max \left(\min \left(\frac{u-\gamma_{1}}{\gamma_{2}-\gamma_{1}}, 1\right), 0\right), \\
& \max \left(\min \left(\frac{u-\gamma_{1}}{\gamma_{2}-\gamma_{1}}, \frac{\gamma_{3}-u}{\gamma_{3}-\gamma_{2}}\right), 0\right) .
\end{aligned}
$$

Consider now the case with a single input linguistic variable $(r=1)$, so that $(8)$ simplifies to

$$
\hat{\mathrm{y}}(\mathrm{t} \mid \boldsymbol{\theta})=\sum_{j=1}^{\mathrm{n}} \alpha_{j} \cdot \mu_{\mathrm{A}_{j}}\left(\varphi(t), \boldsymbol{\beta}_{j}, \gamma_{j}\right) \text {. }
$$

We are here searching for parameter values and MFs guaranteeing that the predictor is monotonically increasing in $\varphi(t)$. This turns out to be a simple task when the input MFs form a fuzzy partition.

To see this, assume that all input MFs are ordered on the universe $\mathbb{U}$ in such a way that $\mu_{A_{j}}(\cdot)$ reaches a full degree of membership for a value of $\varphi(t)$ that is lower than what is the case for $\mu_{A_{j+1}}(\cdot)$. If the ordered MFs at the input side form a fuzzy partition and the corresponding centers $\alpha_{j}$ reflecting the output MFs are such that

$$
\alpha_{1}<\alpha_{2}<\ldots<\alpha_{n}
$$

then $\hat{y}(t \mid \theta)$ will show a monotonically increasing behavior. In verifying this, we first notice that at intervals where the $j$ th input $\mathrm{MF}$ is fully active then the corresponding output becomes $\alpha_{j}$. With fuzzy partitions constructed by piecewise linear MFs we also have that

$$
\begin{aligned}
\hat{y}(t \mid \theta) & =\alpha_{j} \mu_{A_{j}}(\cdot)+\alpha_{j+1} \mu_{A_{j+1}}(\cdot) \\
& =\left(\alpha_{j+1}-\alpha_{j}\right) \mu_{A_{j+1}}(\cdot)+\alpha_{j}
\end{aligned}
$$

for all intervals $\left[\gamma_{j}, \gamma_{j+1}\right] \subset \mathbb{U}$ such that $\mu_{A_{j}}(\cdot)$ and $\mu_{A_{j+1}}(\cdot)$ are not always zero. Since $\alpha_{j+1}>\alpha_{j}$ (equality gives a constant output on the current interval) and $\mu_{A_{j+1}}(\cdot)$ is an increasing function on $\left[\gamma_{j}, \gamma_{j+1}\right]$ it follows that also $\hat{y}(t \mid \theta)$ is an increasing function on that interval, with values ranging from $\alpha_{j}$ to $\alpha_{j+1}$. These facts give that the overall predictor is a non-decreasing function. To get a strictly increasing mapping it additionally must be required that the input MFs lack intervals with a full degree of membership.

The next step is to generalize this result to predictors having $r$ regressors. To do this we start by formally defining what is meant by a monotonically increasing predictor.

Definition 2. (regressor ordering). Let $\varphi(t)$, $\bar{\varphi}(\mathrm{t}) \in \mathbb{R}^{r}$. We say that $\boldsymbol{\varphi}(\mathrm{t}) \geq \bar{\varphi}(\mathrm{t})$ if $\varphi_{\mathrm{k}}(\mathrm{t}) \geq$ $\bar{\varphi}_{k}(\mathrm{t})$ for $\mathrm{k}=1, \ldots, \mathrm{r}$.

Definition 3. (monotonically increasing predictor). Let $\varphi(t), \bar{\varphi}(t) \in \mathbb{R}^{r}$. We say that a predictor $\mathrm{g}(\varphi(\mathrm{t}), \boldsymbol{\theta})$ is monotonically increasing in the regressors if whenever $\varphi(t) \geq \bar{\varphi}(\mathrm{t})$ it holds that $g(\varphi(t), \theta) \geq g(\bar{\varphi}(t), \theta)$.

We now have the following main theorem.

Theorem 1. Let the model structure be complete and given by (8). If, for all $\mathrm{k}=1, \ldots, \mathrm{r}$, it holds that

$$
\sum_{j_{k}=1}^{n_{k}} \alpha_{j} \cdot \mu_{A_{j_{k}, k}}\left(\varphi_{k}(t), \beta_{j_{k}, k}, \gamma_{j_{k}, k}\right)
$$

are increasing functions in $\varphi_{k}(t)$ on $\mathbb{U}_{k}$ for all possible combinations of fixed values of $j_{1}, \ldots$, $\mathfrak{j}_{k-1}, \mathfrak{j}_{k+1}, \ldots, \mathfrak{j}_{\mathfrak{r}}$, then the predictor (8) is monotonically increasing in the regressors $\varphi(t)$.

Proof. Let $\varphi_{\mathrm{l}}(\mathrm{t})$ denote any regressor and fix all other regressors $\varphi_{\mathrm{k}}(\mathrm{t})=\varphi_{\mathrm{k}}^{*} \in \mathbb{U}_{\mathrm{k}}, \mathrm{k}=$ $1, \ldots, l-1, l+1, \ldots, r$. Rearranging the terms 

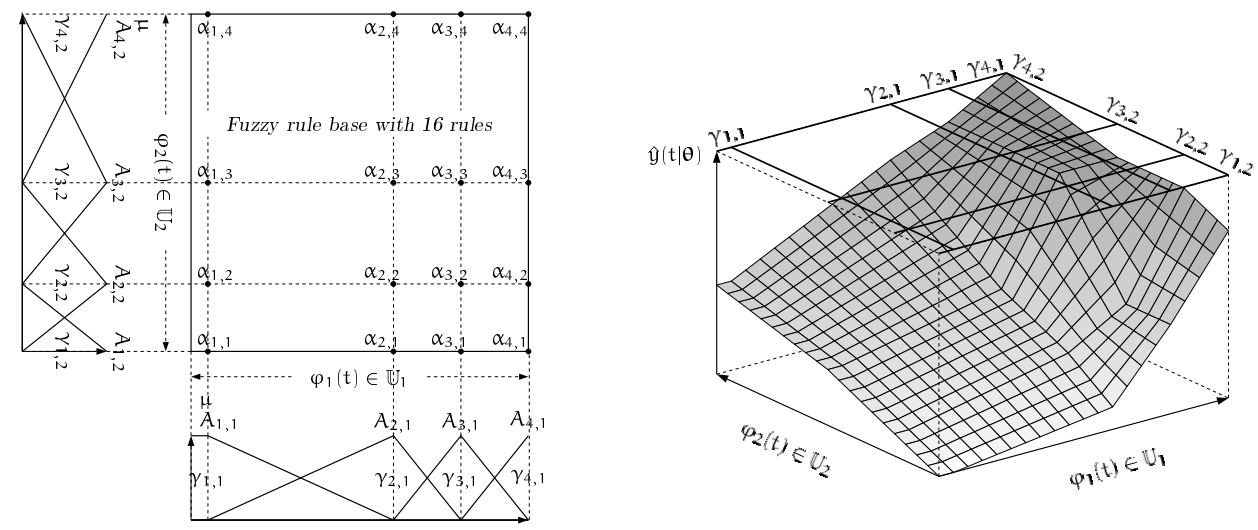

Fig. 2. Graphical representation of a complete fuzzy rule base containing 16 rules (left). Both linguistic variables at the input side have MFs forming fuzzy partitions. Ordering the centers as $\alpha_{j_{1}, j_{2}} \leq$ $\alpha_{j_{1}, j_{2}+1}$ for $j_{1}=1, \ldots, 4, j_{2}=1, \ldots, 3$ and as $\alpha_{j_{1}, j_{2}} \leq \alpha_{j_{1}+1, j_{2}}$ for $j_{1}=1, \ldots, 3, j_{2}=1, \ldots, 4$ gives an increasing mapping as is shown in the right plot.

of the predictor (8) gives

$$
\begin{aligned}
\hat{y}(t \mid \theta)= & \sum_{j_{1}=1}^{n_{1}} \ldots \sum_{j_{l-1}=1}^{n_{l-1}} \sum_{j_{l+1}=1}^{n_{l+1}} \ldots \sum_{j_{r}=1}^{n_{r}} \\
& \prod_{\substack{k=1 \\
k \neq l}}^{r} \mu_{A_{j_{k}, k}}\left(\varphi_{k}^{*}\right) \sum_{j_{l}=1}^{n_{l}} \alpha_{j} \cdot \mu_{A_{j_{l}, l}}\left(\varphi_{l}(t)\right),
\end{aligned}
$$

where for simplicity the $\boldsymbol{\beta}_{\boldsymbol{j}_{\mathrm{k}}, \mathrm{k}}$ and the $\boldsymbol{\gamma}_{\boldsymbol{j}_{\mathrm{k}}, \mathrm{k}}$ parameters have been dropped. The first part of the expression (including the product) returns weights formed by taking the product of $r-1$ MFs, i.e., all the weights lie in $[0,1]$. By the assumption, the last sum returns functions that are increasing in $\varphi_{\mathrm{l}}(\mathrm{t})$ on $\mathbb{U}_{\mathrm{l}}$, which means that the predictor is a weighted (positive weights) sum of increasing functions. This gives that the overall predictor is monotonically increasing in the regressors $\varphi(t)$.

The main point with Theorem 1 is that it is sufficient to work with one-dimensional functions. A simple way to ensure increasing functions in all $\varphi_{k}(t)$ is now to restrict the input MFs to fuzzy partitions and order the corresponding centers as was done in the one-dimensional case.

Lemma 1. Let the model structure be (8) and let $\varphi_{k}(t)$ denote one of its regressors. Assume that the ordered (on $\mathbb{U}_{k}$ ) MFs associated with $\varphi_{\mathrm{k}}(\mathrm{t})$ are piecewise linear and such that they form a fuzzy partition. If, for all combinations of $j_{1}, \ldots, j_{k-1}, j_{k+1}, \ldots, j_{r}$, it holds that

$$
\alpha_{j_{1}, \ldots, j_{k}, \ldots, j_{r}} \leq \alpha_{j_{1}, \ldots, j_{k}+1, \ldots, j_{r}},
$$

$\forall j_{k}=1, \ldots, n_{k}-1$, then every

$$
\sum_{j_{k}=1}^{n_{k}} \alpha_{j} \cdot \mu_{A_{j_{k}, k}}\left(\varphi_{k}(t), \gamma_{j_{k}, k}\right)
$$

is a monotonically increasing function in $\varphi_{k}(t)$.

This lemma follows directly from the one-dimensional case discussed above. The requirements for Theorem 1 to hold are fulfilled if all MFs are chosen according to Lemma 1 . This is the case for the rule base in Fig. 2, from which it is clear that the resulting predictor returns a larger (or unchanged) output if one or more of the regressors become larger. Moreover, if the orders among the parameters $\boldsymbol{\alpha}$ and $\gamma$ are maintained in the estimation step, then this is a fact that cannot be altered by the estimation procedure. Using a squared prediction error optimization criterion, the then obtained constrained minimization problem can be solved, e.g., by a barrier function method; see (Fletcher, 1987) for algorithmic details.

At this point, assume that the regressors include dynamics

$$
\begin{array}{r}
\varphi(t)=[y(t-1) y(t-2) \ldots \\
u(t) u(t-1) \ldots]^{\top},
\end{array}
$$

where, without loss of generality, only one input signal is present. A globally asymptotically stable predictor in $\varphi(t)$ implies that a constant input $\mathrm{u}^{*}=\mathrm{u}(\mathrm{t})=\mathrm{u}(\mathrm{t}-1)=\ldots$ leads to a constant output $y^{*}$ as $t \rightarrow \infty$. Plotting $y^{*}$ for each value of $u^{*}$ gives the steady-state gain curve.

Lemma 2. Let $\mathrm{u}^{*}, \mathrm{y}^{*}$ and $\overline{\mathrm{u}}^{*}, \overline{\mathrm{y}}^{*}$ be two steadystate solutions to a globally asymptotically stable predictor $\mathrm{g}(\boldsymbol{\varphi}(\mathrm{t}), \boldsymbol{\theta})$, i.e.,

$$
\begin{aligned}
& y^{*}=g\left(\left[\begin{array}{lllll}
y^{*} & y^{*} \ldots u^{*} u^{*} \ldots
\end{array}\right]^{\top}, \theta\right), \\
& \bar{y}^{*}=g\left(\left[\begin{array}{llllll}
\bar{y}^{*} & \bar{y}^{*} \ldots & \bar{u}^{*} & \bar{u}^{*} \ldots
\end{array}\right]^{\top}, \theta\right) .
\end{aligned}
$$

If $\mathrm{g}(\varphi(\mathrm{t}), \theta)$ is monotonically increasing in $\varphi(\mathrm{t})$ and $\mathrm{u}^{*} \geq \overline{\mathrm{u}}^{*}$, then $\mathrm{y}^{*} \geq \overline{\mathrm{y}}^{*}$.

Proof. Suppose that $\mathrm{y}^{*}<\overline{\mathrm{y}}^{*}$. Let $\mathrm{u}(\mathrm{t})=\overline{\mathrm{u}}^{*}$ for $\mathrm{t} \leq 0$, whereupon $\mathrm{u}(\mathrm{t})=\mathrm{u}^{*}$ for $\mathrm{t}>0$. This input sequence results in an output sequence $\{\mathrm{y}(\mathrm{t})\}$ and $r$ regressor sequences $\left\{\varphi_{k}(t)\right\}$. If $y^{*}<\bar{y}^{*}$, then there exists a $t^{*}$ such that $y\left(t^{*}\right)<\bar{y}^{*}$ occurs for the first time:

$$
\begin{gathered}
y\left(t^{*}\right)=g\left(\left[y\left(t^{*}-1\right) y\left(t^{*}-2\right) \ldots\right.\right. \\
\left.\left.u^{*} u^{*} \ldots\right]^{\top}, \theta\right)=g\left(\varphi\left(t^{*}\right), \theta\right) .
\end{gathered}
$$

Since this happens for the first time we have that $\varphi\left(\mathrm{t}^{*}\right) \geq \bar{\varphi}^{*}$, which by the monotonicity assumption implies that $\mathrm{y}\left(\mathrm{t}^{*}\right) \geq \overline{\mathrm{y}}^{*}$. Contradiction! 
Thus, if the requirements of Lemma 2 are fulfilled, then we get a predictor with a monotonically increasing steady-state gain curve in the input. Also, starting from a steady-state solution and increasing the input in a stepwise fashion, it follows by simple induction that $\hat{y}(t \mid \theta)$ increases monotonically with $t$. This in particular means that the predictor shows a non-oscillatory step response behavior, which is a restriction but also a property that is valid for many industrial processes (e.g., thermal systems), as will be illustrated next.

\section{EXAMPLE-WATER HEATING SYSTEM}

This section considers identification of a water heating system, depicted in Fig. 3. The process has earlier been investigated by (Koivisto, 1995).

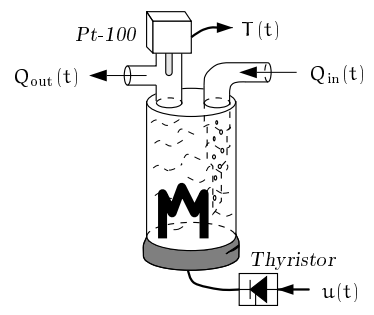

Fig. 3. The water heating process.

Water flows across an uninsulated 0.4 liter tank. On its way, the water is heated by a resistor element, which is controlled by the voltage $u(t)$ applied to a thyristor. At the outlet, the water temperature $\mathrm{T}(\mathrm{t})$ is measured with a $\mathrm{Pt}-100$ transducer. As in (Koivisto, 1995), we will here restrict the discussion to a situation where $Q_{\text {in }}(t)$ as well as the inlet water temperature is constant. The modeling problem is then to describe the outlet water temperature $T(t)$ given the voltage $u(t)$.

The data to be used originate from a real time identification run (performed by Koivisto), where the process was driven by a pseudo-random type of input signal $u(t)$ (given in percent of the maximum allowed voltage). The experiment lasted 9000 secs. and measurements were recorded every 3rd sec. The obtained data set was then divided into an estimation set of 2000 samples and into a validation set of 1000 samples.

Before performing any identification experiments we next list some important properties of the heating system.

1. Step response tests show that the time delay from that a change in the input can be seen in the output is 12 to 15 secs. Since the sampling interval is 3 secs., useful regressors stemming from the input are $u(t-4), u(t-5)$, and so on.

2. The thyristor has a saturating characteristics.

3. The temperature $T(t)$ will increase if more power (a larger $u(t)$ ) is applied to the heater element. The steady-state gain curve of a physically sound model should thus be monotonically increasing in $u(t)=u^{*}$.

The last item is extremely important as the model is going to be used in a model predictive control (MPC) arrangement, see (Koivisto, 1995), where the aim of the control is to drive the temperature $\mathrm{T}(\mathrm{t})$ to a desired set-point value. To explain this, suppose that the current steady-state point lies in an area where the steady-state gain curve is decreasing in $u(t)$. Locally this curve indicates that to decrease the temperature $T(t)$, one should actually increase the voltage $u(t)$. Such a decision is of course fatal as it is known from physics that an increase in the voltage leads to an increase in the outlet temperature. A controller based on this model will thus react in a qualitatively opposite manner to what is reasonable, and in the end cause severe stability problems.

In (Koivisto, 1995) it is stressed that the important monotonicity property easily can be violated if neural network structures are fit to the data without precaution. This problem occurs for large input signals and is due to the lack of identification data at higher temperatures.

This difficulty gives us a reason to try the fuzzy model structure (8). Desiring a model of low complexity, correlation tests indicate that $\mathrm{T}(\mathrm{t}-$ $1)=\varphi_{1}(t)\left(\operatorname{Temp}(t-1) \in \mathbb{U}_{1}=[10,50]\right)$ and $u(t-5)=\varphi_{2}(t)\left(\operatorname{volTage}(t-5) \in \mathbb{U}_{2}=[0,100]\right)$ are reasonable regressor candidates. The listed properties are achieved if the MFs (VL, L, RL, M, $\mathrm{H}$ and $\mathrm{VH}$ are abbreviations for VERY LOW, LOW, RATHER LOW, MEDIUM, HIGH and VERY HIGH)

$$
\begin{aligned}
\mu_{\mathrm{VL}}(\hat{\mathrm{T}}(\mathrm{t} \mid \boldsymbol{\theta})) & =\alpha_{1,1}, \quad \mu_{\mathrm{L}}(\hat{\mathrm{T}}(\mathrm{t} \mid \boldsymbol{\theta}))=\alpha_{1,2}, \\
\mu_{\mathrm{RL}}(\hat{\mathrm{T}}(\mathrm{t} \mid \boldsymbol{\theta})) & =\alpha_{2,1}, \quad \mu_{\mathrm{M}}(\hat{\mathrm{T}}(\mathrm{t} \mid \boldsymbol{\theta}))=\alpha_{2,2}, \\
\mu_{\mathrm{H}}(\hat{\mathrm{T}}(\mathrm{t} \mid \boldsymbol{\theta})) & =\alpha_{3,1}, \quad \mu_{\mathrm{VH}}(\hat{\mathrm{T}}(\mathrm{t} \mid \boldsymbol{\theta}))=\alpha_{3,2},
\end{aligned}
$$

and

$$
\begin{aligned}
& \mu_{A_{1,1}}\left(\varphi_{1}(t), \cdot\right)=\operatorname{mfl}\left(\varphi_{1}(t), \gamma_{1,1}, \gamma_{2,1}\right), \\
& \mu_{A_{2,1}}\left(\varphi_{1}(t), \cdot\right)=\operatorname{mftri}\left(\varphi_{1}(t), \gamma_{1,1}, \gamma_{2,1}, \gamma_{3,1}\right), \\
& \mu_{A_{3,1}}\left(\varphi_{1}(t), \cdot\right)=\operatorname{mfr}\left(\varphi_{1}(t), \gamma_{2,1}, \gamma_{3,1}\right), \\
& \mu_{A_{1,2}}\left(\varphi_{2}(t), \cdot\right)=\operatorname{mfl}\left(\varphi_{2}(t), \gamma_{2,1}, \gamma_{2,2}\right), \\
& \mu_{A_{2,2}}\left(\varphi_{2}(t), \cdot\right)=\operatorname{mfr}\left(\varphi_{2}(t), \gamma_{2,1}, \gamma_{2,2}\right),
\end{aligned}
$$

are used in the predictor

$$
\hat{\mathrm{T}}(\mathrm{t} \mid \boldsymbol{\theta})=\sum_{j_{1}=1}^{3} \sum_{j_{2}=1}^{2} \alpha_{j_{1}, j_{2}} \prod_{k=1}^{2} \mu_{A_{j_{k}, k}}(\cdot),
$$

which contains 11 parameters

$$
\begin{gathered}
\theta=\left[\begin{array}{cccccc}
\alpha_{1,1} & \alpha_{1,2} & \alpha_{2,1} & \alpha_{2,2} & \alpha_{3,1} & \alpha_{3,2} \\
\gamma_{1,1} & \gamma_{2,1} & \gamma_{3,1} & \gamma_{1,2} & \gamma_{2,2}
\end{array}\right]^{\top}
\end{gathered}
$$

chosen so that

$$
\begin{aligned}
10 & <\alpha_{1,1}<\alpha_{1,2}<\alpha_{2,1} \\
& <\alpha_{2,2}<\alpha_{3,1}<\alpha_{3,2}<50 \\
10 & <\gamma_{1,1}<\gamma_{2,1}<\gamma_{3,1}<50 \\
0 & <\gamma_{1,2}<\gamma_{2,2}<100 .
\end{aligned}
$$

A graphical representation of the corresponding fuzzy rule base is shown in Fig. 4, where dotted lines represent the initial positions of the MFs. Constrained estimation of $\theta$ subject to the constraints (18) results in a model (with MFs according to Fig. 4), whose simulation behavior is reproduced in Fig. 5. 

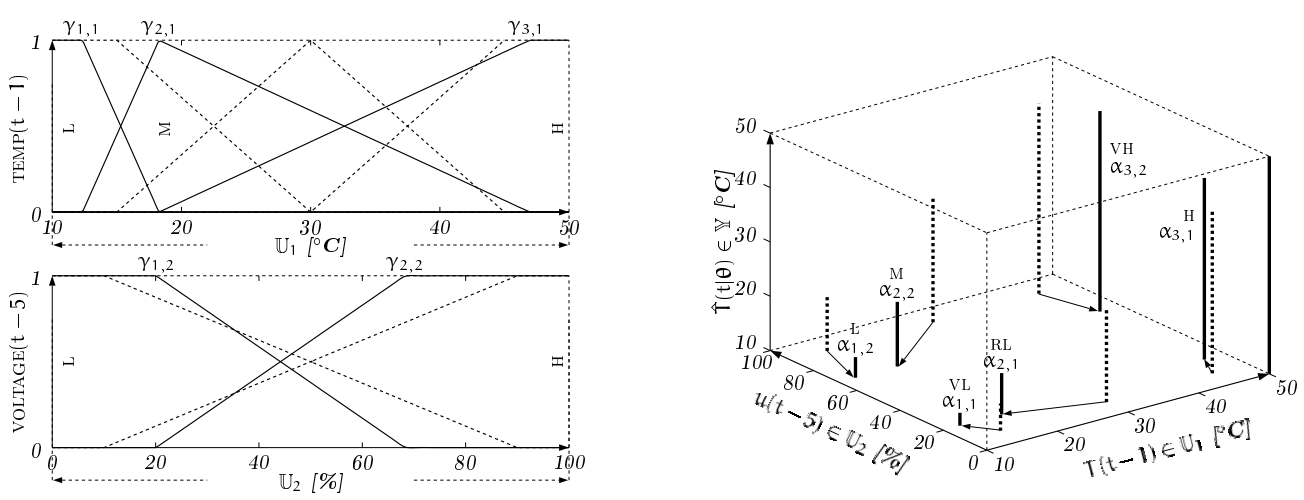

Fig. 4. Input (left) and output (right) MFs used to describe the temperature of the heating system. Dotted and solid curves show the situation before and after estimation, respectively.

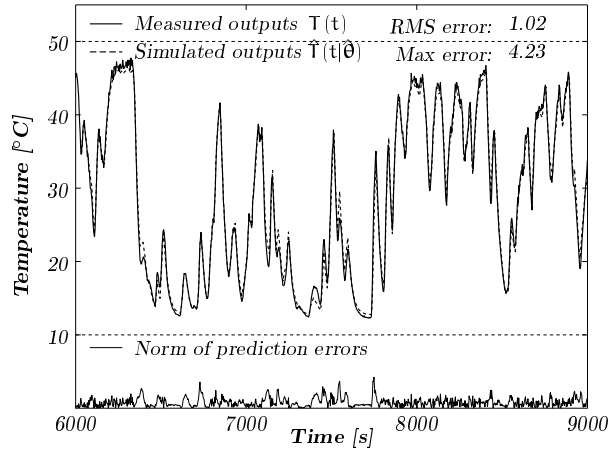

Fig. 5. Simulation of the fuzzy heating model.

Compared to the best linear model found (an ARX model with four parameters), the root mean square (RMS) error decreases from 2.08 to 1.02 , i.e., it is halved. The improvement is significant at low and high temperatures, which mainly is due to that the linear model cannot capture the saturating characteristics of the thyristor. The built-in increasing nature of the steady-state gain curve of this model is shown in Fig. 6 .

From these experiments we conclude that the derived fuzzy model is able to accurately describe the water heating system, at the same time as the important monotonicity property is ensured. The obtained RMS error 1.02 should be compared to 0.92 , which is obtained in (Koivisto, 1995) using a neural net having many more parameters (31 compared to 11 in the fuzzy case). This along with the ensured monotone behavior suggest that the fuzzy approach might be a good alternative to neural nets when applied to predictive control.

\section{CONCLUSION}

We have in this paper addressed the problem of "dark grey boxes": How to include, ensure and maintain a known qualitative behavior of a process in a model structure that is otherwise quite flexible. This is difficult to achieve in traditional grey box structures, unless a rather precise physical knowledge in analytic form is at hand.

To deal with the problem, we have turned to black box structures of the kind (1), using basis func-

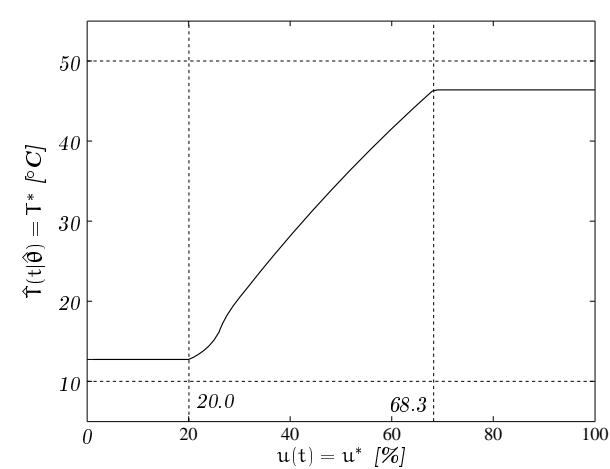

Fig. 6. Steady-state gain curve of the obtained fuzzy heating model.

tions $g_{j}(\cdot)$ that have some useful interpretation related to the system's behavior. This is the basic property of fuzzy models. We have shown how to parameterize these basis functions ("membership functions") and how to constrain the parameters so that certain monotonicity properties in the model's response are guaranteed.

Real-world experiments - in this case Koivisto's heating system - as well as other applications have demonstrated the usefulness of the approach.

\section{REFERENCES}

Driankov, D., H. Hellendoorn and M. Reinfrank (1993). An Introduction to Fuzzy Control. Springer-Verlag.

Fletcher, R. (1987). Practical Methods of Optimization. John Wiley \& Sons.

Koivisto, H. (1995). A Practical Approach to Model Based Neural Network Control. Phd thesis 170 . Tampere University of Technology. Tampere, Finland.

Lindskog, P. (1996). Methods, Algorithms and Tools for System Identification Based on Prior Knowledge. Phd thesis 436. Department of Electrical Engineering, Linköping University. Linköping, Sweden.

Sjöberg, J., Q. Zhang, L. Ljung, A. Benveniste, B. Delyon, P.-Y. Glorennec, H. Hjalmarsson and A. Juditsky (1995). Nonlinear black-box modeling in system identification: a unified overview. Automatica 31(12), 1691-1724. 